\title{
Spectral Enhancement of a SiPM Array-Based Radiation Detector
}

\author{
R. Harn ${ }^{1,3, *}$, A. Osovizky ${ }^{1,2}$, Y. Kadmon ${ }^{1}$, S. Rotman ${ }^{3}$, N. Kopeika ${ }^{3}$, and M. Ghelman ${ }^{1,3}$ \\ ${ }^{1}$ Nuclear Research Center - Negev, Israel \\ ${ }^{2}$ Radiation Detection Department, Rotem Industries Ltd, Israel \\ ${ }^{3}$ Ben-Gurion University, School of Electrical and Computer Engineering, Israel \\ *RonH@nren.org.il
}

\begin{abstract}
Silicon Photomultipliers (SiPMs) have many advantages when used in radiation detectors. Low bias voltage, compactness and immunity to electromagnetic interference are among their prominent benefits. However, due to their small size, usually an array of SiPM components is required in order to cover the coupling surface area of a scintillator. Since the SiPM is a semiconductor, biased in a reversed voltage, gain variation and strong temperature dependence are introduced. As a result, SiPM-based detectors, particularly an array of SiPMs, undergo spectral signal to noise ratio reduction. This work studies the effect of the SiPM breakdown voltage variation on the obtained energy spectrum and proposes an electronic approach to overcome this technological drawback. This developed technology provides an adequate temperature-dependent, commonly distributed high bias voltage and an individual offset-voltage fine tuning that enables adjustment of all the SiPM components to their optimum operating points. Power-wise it is beneficial to operate SiPM at lower voltages, where undesirable gain variation is more dominant. The proposed solution enables working at lower bias voltages, which provides lower power consumption and better radiation hardness, while yielding an enhanced spectrum resolution. The proposed electronic approach enhances the obtained spectra, reducing the noise threshold by $16 \%$ when working at $1 \mathrm{~V}$ overvoltage. Hence provides an enhanced signal to noise ratio over the traditional biasing methods.
\end{abstract}

Keywords - SiPM, Array, Radiation detectors, SNR, Breakdown Voltage.

\section{INTRODUCTION}

$\mathrm{R}$ ADIATION detectors based on arrays of silicon photomultipliers (SiPMs) coupled to a light scintillator are becoming more and more popular, gradually replacing traditional photomultiplier tube (PMT) based detectors [1]. Among their many uses, SiPM based detectors can be found in medical imaging devices such as positron emission tomography (PET) [2] and single photon emission tomography (SPECT) [3], homeland security applications [4], environmental monitoring with drones [5], and particle astrophysics experiments with radiation detector mounted on a satellite [6,7]. Its compactness, relatively low bias voltage and immunity to electro-magnetic interference (EMI) give the SiPM superiority over the traditional PMT in radiation detector implementations, especially for the two last applications mentioned above.

The gain of the SiPM is voltage dependent. The overvoltage, $V_{O V}$, is defined as the difference between the supplied bias voltage, $V_{B}$, and the SiPMs breakdown voltage, $V_{B D}$, as shown in (1),

$$
V_{O V}=V_{B}-V_{B D}
$$

For low $V_{O V}$, the SiPM amplification rises linearly with $V_{O V}$. The avalanche initiation probability (AIP) increases linearly together with $V_{O V}$ as well. Hence, the SiPMs total light detection gain has a parabolic dependence on $V_{O V}$. Despite the lower obtained gain, using lower $V_{O V}$ can provide advantages such as power consumption reduction and radiation hardness $[8,9]$, both of which are crucial for radiation detectors mounted on satellites and exposed to cosmic ray radiation, and for drones scanning polluted areas with high radiation fields.

Nevertheless, SiPMs have two fundamental drawbacks compared to the PMT. First, the SiPM breakdown voltage has a strong temperature dependence, which leads to gain dependent on the temperature, and second, variation in the breakdown voltage as a result of the limitations of the manufacturing process. The breakdown voltage temperature dependence was widely studied in previous works $[10,11]$ and several solutions were presented to address it $[12,13]$. However, the breakdown voltage variation, that result from fluctuations in the resistivity of the epitaxial layer that hosts the SiPM active region [11], and its effect on the obtained spectra were less studied. This variation results in gain fluctuation between SiPM components in the same array and a reduction of the total signal to noise ratio (SNR) obtained from the detector at lower voltages. The reduced SNR is an outcome of the photon detection efficiency (PDE) curve shape [14], which rises more moderately as the overvoltage increases. Hence, a reduction of $\Delta V$ in the overvoltage, due to the breakdown variation, will decrease the PDE more significantly in respect to the increase of the PDE as a result of a growth of the overvoltage in the same $\Delta V$. The result is a reduction of the average PDE value for an array of SiPM components as compared to the average PDE of an ideal homogeneous SiPM array. This reduction will be expressed in a lower number of fired pixels and therefore lower statistical significance.

Over the years, several approaches for addressing the breakdown voltage variation have been introduced. One approach is to provide a separate adjustable gain preamplifier for each SiPM [15]. Although this approach 
provides a uniform gain from all SiPM readouts, it does not address the reduction in the obtained SNR. Moreover, due to the SiPM pulse shape, high bandwidth amplifiers would be needed, hence increasing the power consumption and the electronic noise. Another approach is to provide a separate temperature dependent bias voltage to each SiPM separately. Even though this approach compensates for the breakdown voltage variation and its temperature dependence, it is very inefficient in power consumption, electronics size, and cost, especially for large SiPM arrays. In this paper, we propose an electronic approach to overcome this technological drawback.

\section{BREAKDOWN VOLTAGE VARIATION INVESTIGATION}

Various SiPM manufacturers possess different production technologies, hence providing SiPM components with different temperature and breakdown voltage variation characteristics. Table I summarizes the temperature dependence and variation of the breakdown voltages of three leading SiPM manufacturers.

TABLE I

CHARACTERISTICS OF SiPMS OF LEADING MANUFACTURERS

\begin{tabular}{lccc}
\hline \hline \multicolumn{1}{c}{ Property } & On Semi. & Ketek & Hamamatsu \\
& {$[16,17]$} & {$[18]$} & {$[19]$} \\
\hline Series & $\mathrm{C}-30050$ & PM3347-WL & S14160-3050 \\
AVG gain & $6 \times 10^{6}$ & $7 \times 10^{6}$ & $2.5 \times 10^{6}$ \\
MAX PDE (\%) & 47 & 51 & 50 \\
AVG $\mathrm{V}_{\mathrm{BD}}(\mathrm{V})$ & 24.4 & 29.5 & 38 \\
$\mathrm{STD}\left(\mathrm{V}_{\mathrm{BD}}\right)(\mathrm{mV})$ & 73 & 92 & 100 \\
Vov range $(\mathrm{V})$ & $1-5$ & $1-6.5$ & $2-7$ \\
$\mathrm{STD}\left(\mathrm{V}_{\mathrm{BD}}\right) / \mathrm{Vov}(\%)$ & 2.43 & 3.33 & 2.2 \\
$\Delta \mathrm{V}_{\mathrm{BD}} / \Delta \mathrm{T}\left(\mathrm{mV} /{ }^{\circ} \mathrm{C}\right)$ & 21.5 & 22 & 34 \\
$\Delta \mathrm{V}_{\mathrm{BD}} / \mathrm{Vov}(\%)$ & 0.7 & 0.58 & 0.75 \\
\hline \hline
\end{tabular}

$\mathrm{AVG}=$ Average, $\mathrm{V}_{\mathrm{BD}}=$ Breakdown voltage, $\mathrm{STD}=$ Standard deviation, Vov $=$ Overvoltage, $\mathrm{TMP}=$ Temperature.

As can be seen in Table I, SiPM components from different manufacturers vary in their breakdown voltages, their overvoltage range, and their temperature dependence. However, all of them have a variation in the breakdown voltage that is not negligible.

In order to implement a $2 \times 2 \mathrm{SiPM}$ array detector, the current curves of $10 \mathrm{C}$-series $60035 \mathrm{SiPM}$ components [16], were measured as a function of the bias voltage. The curves obtained are presented in Fig. 1. The breakdown voltage of the $10 \mathrm{SiPM}$ components was obtained from the current measurement using the inverse logarithmic derivative (ILD) method [20]. The usefulness of this method is that it cancels out constant parameters that affect the current level, such as the number of incoming photons, and yields a linear dependence in $V_{O V}$ in the near breakdown voltages. Hence, by using (2) to calculate the ILD, and finding the intersection of the linear fit for over breakdown voltages, with the voltage axis, we can obtain the measured SiPMs breakdown voltages.

$$
I L D(V)=\left(\frac{d}{d V} \ln [I(V)]\right)^{-1} .
$$

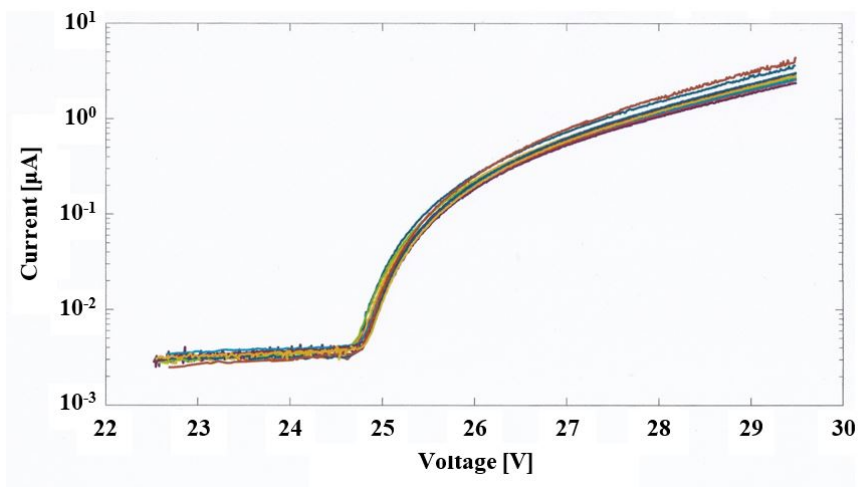

Fig. 1. I-V curve as a function of the bias voltage of ten C-series SiPM components.

The ILD curve and the linear fit of the near breakdown voltages are shown in Fig. 2. The breakdown voltages that were obtained are presented in Fig. 3 over a normal probability density function plotted using the data for the C30050 series breakdown variation from Table I.

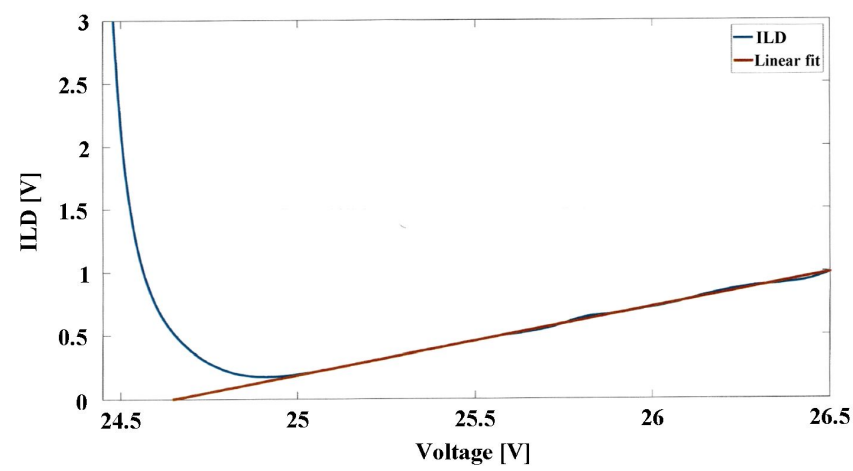

Fig. 2. ILD curve and the linear fit intersect with the voltage axis to yield the breakdown voltage.

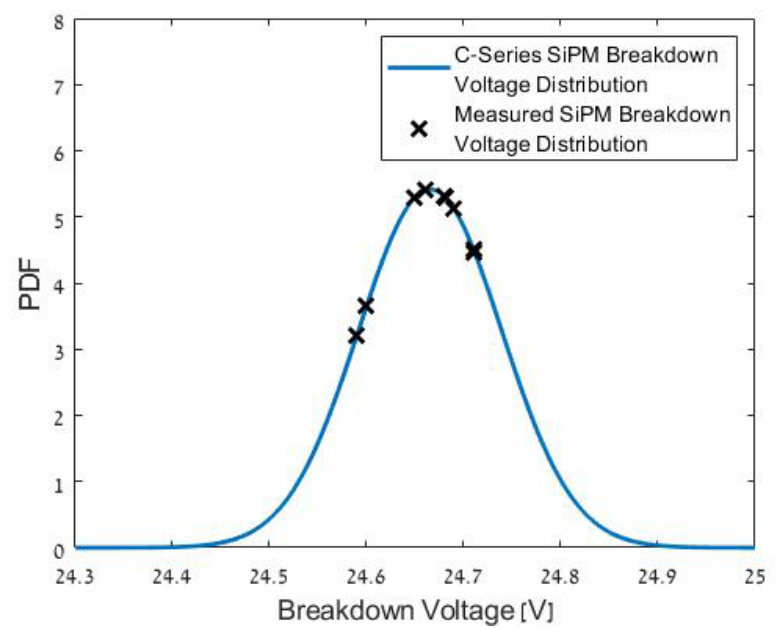

Fig. 3. Ten breakdown voltages that were calculated marked on the normal probability density function plotted using the standard deviation from Table I.

The average voltage that was obtained from the measurements is shifted from the manufacturer average voltage. This is an expected result due to the temperature differences of the measurements and the breakdown voltage dependence of $21^{1 / 2} \mathrm{mV} /{ }^{\circ} \mathrm{C}$ [16]. All the obtained breakdown voltages are in the single standard deviation (STD) interval. 


\section{BREAKDOWN VARIATION CORRECTION TOPOLOGY}

A scintillator detector based on a $2 \times 2$ SiPM array coupled to a $15 \mathrm{~mm} \times 15 \mathrm{~mm} \times 15 \mathrm{~mm} \mathrm{Cs(Tl)}$ scintillator was implemented. The breakdown voltages of the SiPMs assemble the array are listed in Table II. Each SiPM output is loaded with a $200 \Omega$ resistor in order to obtain an equivalent $50 \Omega$ load for all the SiPMs in the array.

TABLE II

SIPM ARRAY BREAKDOWN VOLTAGES

\begin{tabular}{ccc}
\hline \hline Component & $\begin{array}{c}\text { Measured Breakdown } \\
\text { Voltage }(\mathrm{V})\end{array}$ & $\Delta \mathrm{V}$ from AVG $(\mathrm{mV})$ \\
\hline SiPM 1 & 24.71 & +57 \\
SiPM 2 & 24.59 & -62 \\
SiPM 3 & 24.60 & -52 \\
SiPM 4 & 24.71 & +57 \\
\hline \hline
\end{tabular}

In order to overcome the breakdown voltage variation, an offset voltage topology was implemented and connected to the SiPM load as shown in Fig. 4. Each SiPM in the array was connected to a separate offset topology while all the SiPMs were fed from a single temperature dependent bias voltage. The SiPMs output is fed through a coupling capacitor to a single shaping channel which sums the 4 SiPM outputs.

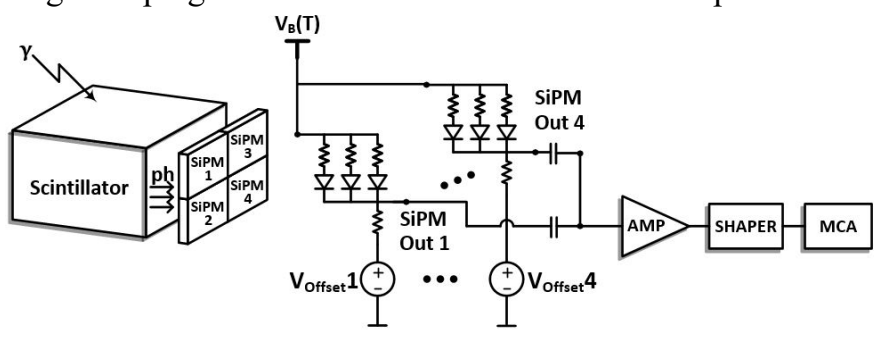

Fig. 4. Simplified block diagram of the proposed detector topology.

A simple approach of implementing the offset voltage would be by using wideband, high power consumption amplifiers that would lead both $\mathrm{AC}$ and DC tracks. In order to reduce power consumption, the suggested topology implements an accurate DC path using narrowband, low power amplifiers, and an AC path through a capacitor, $C_{O f f s e t}$, as shown in Fig. 5. The offset value is set by using a voltage divider or by micro-controller DAC output. Due to the use of narrow bandwidth amplifiers the increase in the electronic noise is minor. The stabilization of the offset amplifier for the capacitive load drive was implemented using the in loop compensation method. In this way the DC feedback comes from the capacitive load itself whereas the AC feedback is fed from the amplifier output through $C f$, bypassing $R s$ and $R f$. The values of $R s, R f$, and $C f$ were calculated according to $C_{\text {Offset }}$ value in order to cancel the additional phase shift added to the amplifier's loop gain as a result of the capacitive load. In this way, the capacitive load can be driven without adding a DC error due to Rs. The supplied bias voltage to each SiPM is the difference between the bias temperature dependent voltage, $V_{B}(T)$, and the SiPMs offset voltage.

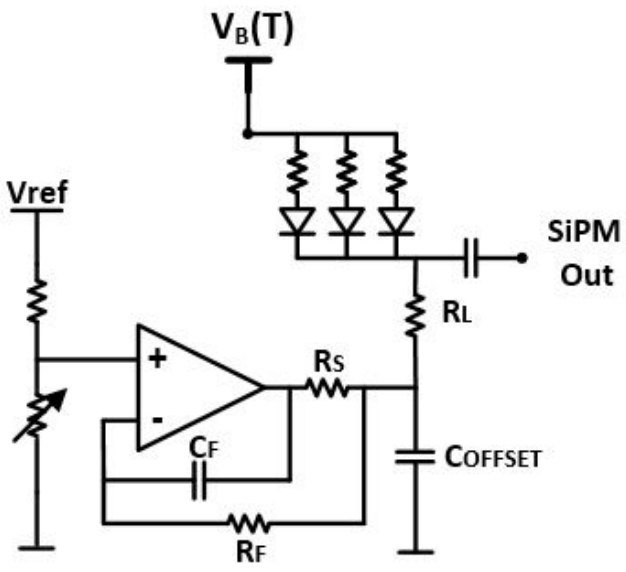

Fig. 5. Offset adjustment topology.

The temperature-dependent bias voltage can be implemented as shown in Fig. 6. The voltage is regulated by adding an accurate temperature sensor to the DC-DC boost converter feedback loop. Hence, the bias voltage is continuously regulated without involving a separate sampling process. The voltage in the boost feedback input is a superposition of the reference voltage and the temperature sensor voltage. By adjusting the values of resistors $R a$ and $R b$ a linear temperature dependence can be implemented.

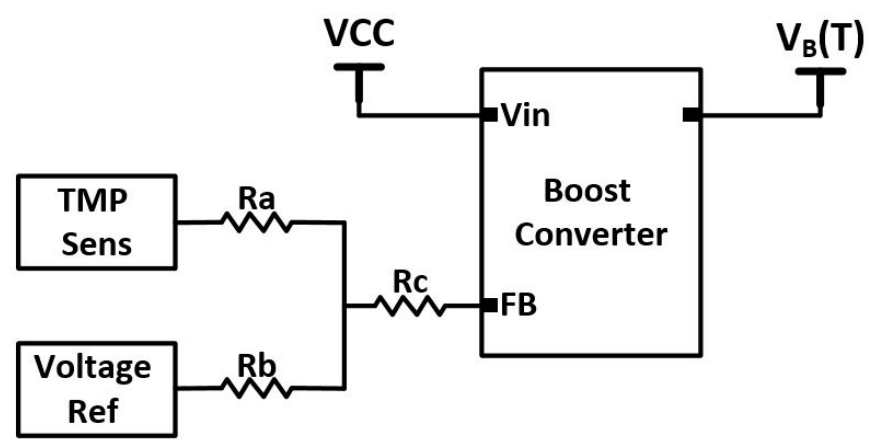

Fig. 6. Temperature dependent bias voltage topology.

\section{MEASurement And ANALysis}

In order to verify that the offset values obtained from the breakdown voltage calculation are the optimal values in SNR perspective, a voltage sweep for each SiPM offset voltage was conducted. At each offset value, the noise threshold was measured while the overall gain remained constant by adjusting the bias voltage of the remaining three SiPMs. This measurement yields a curve of the noise threshold as a function of the offset voltage for each SiPM separately, as shown in Fig. 7. The gap between the average breakdown voltage and the value calculated using the ILD method, $\Delta V$, listed in Table II, is transformed to the correlating offset voltage by taking $\Delta V$ negative value. The offset values that were calculated using the ILD method are marked as ' $\mathrm{X}$ ' on the curves. It can be seen that there is a good agreement between the measured optimal offset and the calculated one. A connection scheme and an image of the measuring setup are shown in Figs. 8 and 9. 

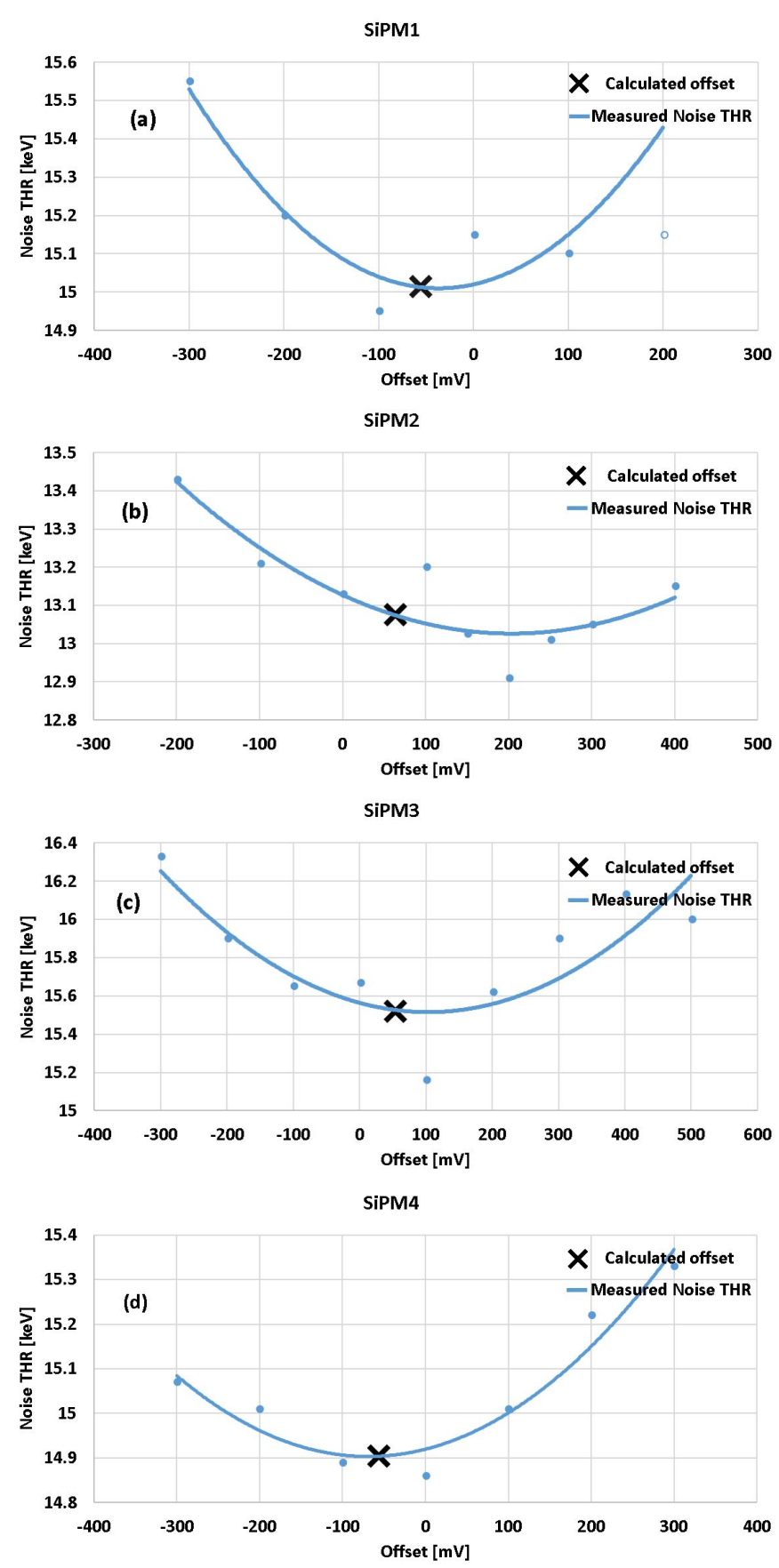

Fig. 7.Noise threshold as a function of the offset voltage for SiPM1 (a), SiPM2 (b), SiPM3 (c), and SiPM4 (d). The 'X' marks the correlating value from Table II.

Temperature Stabilization Chamber

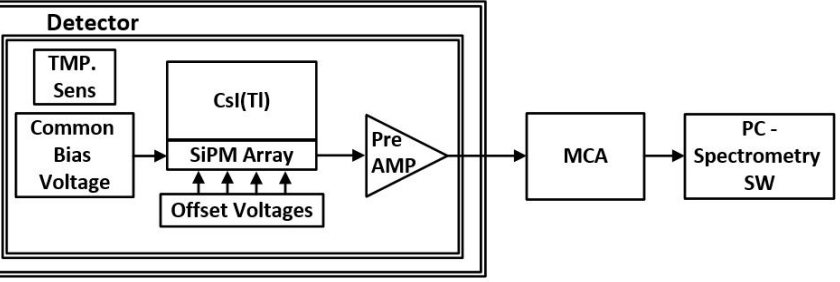

Fig. 8. Connection scheme of the detector and the measuring setup.

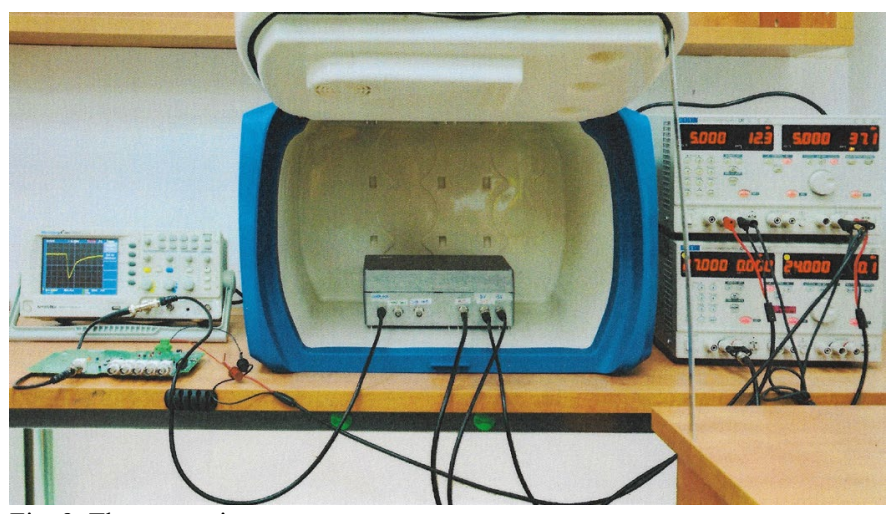

Fig. 9. The measuring setup.

At the second stage, the noise threshold as a function of the $V_{O V}$ was obtained for a $V_{O V}$ interval of $750 \mathrm{mV}-1.75 \mathrm{~V}$. At each voltage, a calibration of the operating point was made followed by a background measurement. The measurement was conducted twice. First, with a uniform voltage offset, hence without the correction of the breakdown voltage variation, and second, with the suggested offset voltage topology based on the values from Table II. In both measurements there was careful attention for the resemblance of the operating point, especially in gain and temperature perspective. The noise threshold was set to obtain 15 counts per second (CPS) above the threshold. Then the noise threshold location on the energy axes was recorded. Assuming normal distribution, the error interval was taken as the square root of the counts number above the threshold. Three STD were taken in each direction and the correlating energy threshold was recorded. The obtained noise threshold curve as a function of $V_{O V}$ without the topology, "Regular", and with the proposed adjustment topology, "ADJ", is shown in Fig. 10.

\section{RESULTS}

As expected, improvement is seen primarily for lower $V_{O V}$ below $1.5 \mathrm{~V}$, as a result of the moderation in the PDE slope for higher $V_{O V}$.

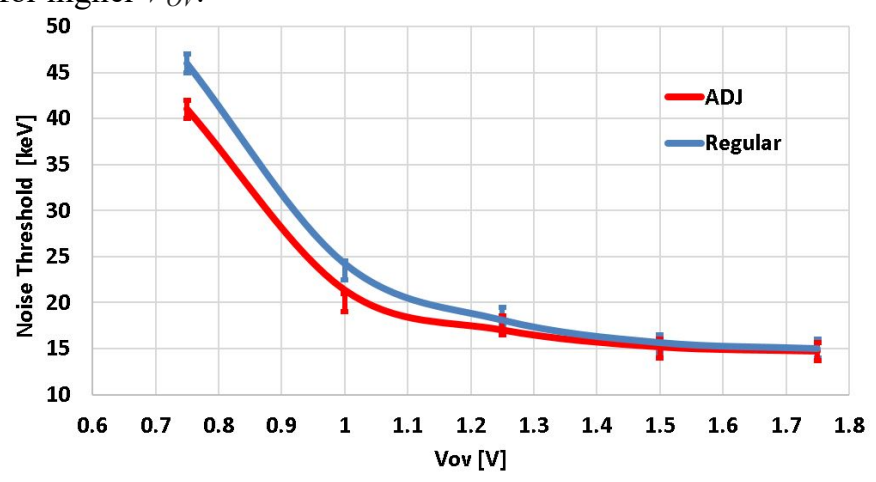

Fig. 10. Noise threshold curve as a function of Vov without the presented topology, "Regular", and with the suggested topology, "ADJ".

As can be seen from Fig. 10, for $1 \mathrm{~V}$ overvoltage the noise threshold was reduced from $24 \mathrm{keV}$ to $20 \mathrm{keV}$, providing 16 $\%$ improvement. A ${ }^{137} \mathrm{Cs}$ spectrum was obtained with, and without the adjustment topology is shown in Fig. 11. The spectrum was obtained using $1 \mathrm{~V}$ overvoltage. As can be seen, when observing the spectra on a wide scale, the difference of the obtained spectra with and without the suggested topology is not significant. However, when focusing on the low energy interval we note that the noise 
peak is shifted to the left in the "ADJ" curve (red), as compared to the spectra without the suggested topology in the "Regular" curve (blue).

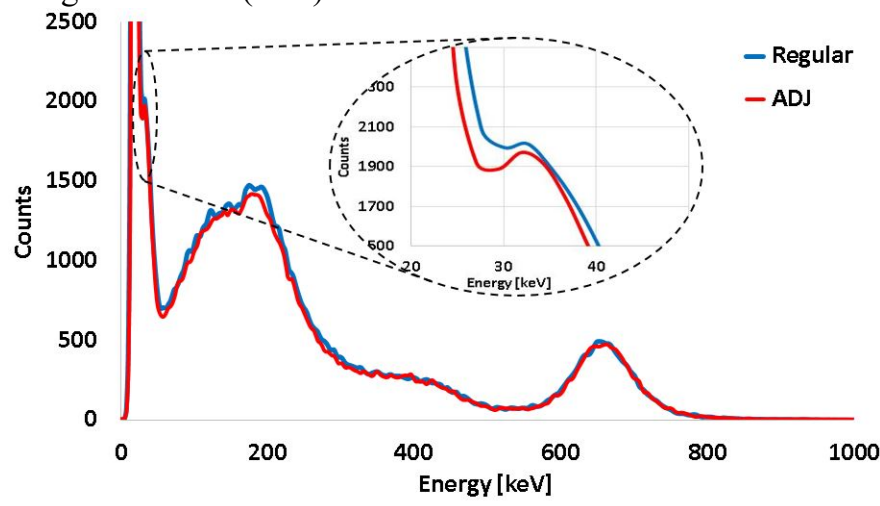

Fig. 11. Spectrum of ${ }^{137} \mathrm{Cs}$ obtained without the use of the breakdown correction topology, "Regular", in blue, and with the topology, "ADJ", in red.

Moreover, while the $32 \mathrm{keV}$ peak is not recognizable in the "Regular" curve, in the "ADJ" curve obtained by the suggested topology the peak is slightly more distinct. Furthermore, the proposed biasing topology is power efficient compared to the straightforward approach of providing a separate bias voltage to each SiPM separately. For comparison, a $\mathrm{n} \times \mathrm{n}$ SiPM array with the proposed topology will have a power consumption as shown in (3) where a separate bias voltage approach will consume as shown in (4).

$$
\begin{gathered}
P_{\text {Adj }}=V_{I n} \cdot I_{Q}+\frac{1}{\eta_{A d j}} \cdot V_{B} \cdot I_{S i P M} \cdot n^{2}, \\
P_{\text {Regular }}=\left(V_{I n} \cdot I_{Q}+\frac{1}{\eta_{\text {Reg }}} \cdot V_{B} \cdot I_{S i P M}\right) \cdot n^{2} .
\end{gathered}
$$

$P_{\text {Adj }}$ and $P_{\text {Regular }}$ are the power consumption of the SiPM array when biased with the proposed adjustment topology and in the regular separate bias voltage approach, respectively. The DC-to-DC converter input voltage is $V_{I n}, I_{Q}$ is the converters supply current, $V_{B}$ is the voltage converters output voltage, and $I_{S i P M}$ is the SiPM current. The DC-to-DC converter efficiency for the proposed topology and for the separate bias voltage are $\eta_{A d j}$ and $\eta_{R e g}$, respectively. From (3) and (4) it can be seen, that when using a separate bias voltage approach the supply current for the DC-to-DC converter is $\mathrm{n}^{2}$ times bigger. Additionally, the DC-to-DC converter efficiency rises as the output current increases, especially for low output currents such as the SiPM DC current. Hence, the DC-to-DC efficiency in the suggested topology, $\eta_{A d j}$, is expected to be significantly bigger than in the separate bias voltage approach.

\section{CONCLUSIONS}

This work investigated the breakdown variation effect on the spectrum that is obtained from SiPM array-based radiation detectors. An electronic topology that addresses the breakdown variation issue and uses a single temperature dependent bias voltage was presented. An improvement in the noise threshold was observed for overvoltages smaller than $1.5 \mathrm{~V}$. Hence, the suggested topology can be useful in cases where a low working voltage can be advantageous. One example is radiation detectors mounted on satellites and exposed to cosmic rays. The SiPMs at such a detector are exposed to proton particle radiation that causes an additional deviation in the breakdown voltage and an increase of the dark current $[7,8]$. Working at $1 \mathrm{~V}$ overvoltage can reduce the dark current by a factor of 20 , compared to standard $3.6 \mathrm{~V}$ overvoltage, at a cost of increasing the SiPM sensitivity to the breakdown voltage variation [21]. Hence, the dark current can be reduced while the breakdown voltage variation is addressed using the proposed topology. Moreover, the proposed topology can correct the mentioned deviation in the breakdown voltage, due to the radiation damage, as a part of a periodic calibration process. Further studies showed that there is a correlation between the working voltage of the SiPM and the effect of the radiation on the SNR and resolution obtained by the detector [9]. Hence, working at low overvoltages and combining the suggested topology can yield a reduced influence of the cosmic rays, provide decreased power consumption, and improved SNR, providing an enhanced spectroscopic preformance.

\section{REFERENCES}

[1] Peter R. Menge, "Efficient positioning of silicon photomultipliers on large scintillation crystals", 2016 IEEE Nuclear Science Symposium, Medical Imaging Conference and Room-Temperature Semiconductor Detector Workshop (NSS/MIC/RTSD), Nov. 2016, 10.1109/NSSMIC.2016.8069734.

[2] Dennis R. Schaart, "A novel, SiPM-array-based, monolithic scintillator detector for PET", Physics in Medicine \& Biology, Vol. 54, Number 11, May 2009, 10.1088/0031-9155/54/11/015.

[3] Paolo Busca, "Experimental Evaluation of a SiPM-Based Scintillation Detector for MR-Compatible SPECT Systems", IEEE Tran. on Nuclear Science, Vol. 62/5, Oct. 2015, 10.1109/TNS.2015.2481184.

[4] Hye MinPark, "Performance characteristics of a silicon photomultiplier based compact radiation detector for Homeland Security applications", Nuclear Instruments and Methods in Physics Research Section A, Vol. 781, May 2015, 10.1016/j.nima.2015.01.080.

[5] Marco Carminati, "A Lightweight SiPM-Based Gamma-Ray Spectrometer for Environmental Monitoring with Drones", presented at the International Conference on Applications in Electronics Pervading Industry, Environment and Society, Lisbon, Jan. 2021.

[6] P. Lv, "A low-energy sensitive compact gamma-ray detector based on LaBr3 and SiPM for GECAM", Journal of Instrumentation, Vol. 13, Aug. 2018, 10.1088/1748-0221/13/08/P08014.

[7] Jason Link, "SiPM Use in Future Space Instruments", presented at the 35th International Cosmic Ray Conference, Korea, Aug. 2018.

[8] Yu. Musienko, " Radiation damage studies of silicon photomultipliers for the CMS HCAL phase I upgrade", Nuclear Instruments and Methods in Physics Research Section A, Vol. 787, pp. 319-322, July 2015, 10.1016/j.nima.2015.01.012.

[9] S. Mianowski, "SiPM proton irradiation for application in cosmic space", Journal of instrumentation, Vol. 15, Mar. 2020, 10.1088/1748-0221/15/03/P03002.

[10] N.Dinu, "Breakdown voltage and triggering probability of SiPM from IV curves at different temperatures", Nuclear Instruments and Methods in Physics Research Section A, Vol. 845, pp. 64-68, Feb. 2017, 10.1016/j.nima.2016.05.110.

[11] Nicola Serra, "Experimental and TCAD Study of Breakdown Voltage Temperature Behavior in n/p SiPMs", IEEE Tran. on Nuclear Science, Vol. 58/3, Jun. 2011, 10.1109/TNS.2011.2123919.

[12] Zhengwei Li, "A gain control and stabilization technique for Silicon Photomultipliers in low-light-level applications around room temperature", Nuclear Instruments and Methods in Physics Research Section A, Vol. 695, pp. 222-225, Dec. 2012, 10.1016/j.nima.2011.12.037.

[13] Seiichi Yamamoto, "A temperature-dependent gain control system for improving the stability of Si-PM-based PET systems", Physics in Medicine \& Biology, Vol. 56, no. 9, pp. 2873-2882, Apr. 2011, 10.1088/0031-9155/56/9/015.

[14] G. Gallina, "Characterization of SiPM Avalanche Triggering Probabilities", IEEE Tran. on Nuclear Science, Vol. 66/10, Oct. 2019, 10.1109/TED.2019.2935690.

[15] Y. C. Shih, "An $8 \times 8$ Row-Column Summing Readout Electronics for Preclinical Positron Emission Tomography Scanners", in Proc. IEEE Nucl Sci Symp Conf., Orlando, FL, USA, 2009, pp. 2376-2380, 10.1109/NSSMIC

[16] ON Semiconductor, "Silicon Photomultipliers (SiPM), Low-Noise, 
Blue-Sensitive", C-Series SiPM Sensors datasheet, May 2021, www.onsemi.com.

[17] ON Semiconductor, "How to Evaluate and Compare Silicon Photomultiplier Sensors", White paper, Oct. 2015.

[18] Ketek, " SiPM - Silicon Photomultiplier", WL-Series datasheet, 2020, www.ketek.net.

[19] Hamamatsu, "Low breakdown voltage type MPPC for scintillation detector", S14160/S14161 Series datasheet, Jun. 2020, www.hamamatsu.com.

[20] V. Chmill, "Study of the breakdown voltage of SiPMs", Nuclear Instruments and Methods in Physics Research Section A, Vol. 845, pp. 56-59, Feb. 2017, 10.1016/j.nima.2016.04.047.

[21] Alexei Ulyanov, "Radiation damage study of SensL J-series silicon photomultipliers using 101.4 MeV protons", Nuclear Instruments and Methods in Physics Research Section A, Vol. 976 , Oct. 2020, 10.1016/j.nima.2020.164203. 